\title{
A Translation Principle Study of English Product Instruction from the Perspective of the Skopos Theory
}

\author{
Jian Wang \\ Zaozhuang University, Zaozhuang, China \\ blood@msn.cn
}

Keywords: Instruction; Localization; Functionalism; Skopos

\begin{abstract}
As international trade and multinational companies have a further development, how to translate product instruction from the source language to the target language consumers to realize the original meaning and function deserves special attention. This paper tries to study the translation principles of products instructions from the perspective of the Skopos Theory, which is the core of the Functionalism. This paper chooses "equifunctional" translation as instruction strategy, and then puts forward three translation principles, hopefully, which will help the translation of product instructions.
\end{abstract}

\section{Introduction}

With the development of economy globalization, it is commonly accepted hat no country can sustain itself in the $21^{\text {st }}$ century. Globalization promotes international trade with the free trade policy between countries. As an important attachment to a product, a well-localized product instruction translation satisfy the overseas' consumers and enhance brand loyalty. As a category of translation, instruction translation does not have right attention it deserves in China. Many outsourcing translation of product instruction only gives the basic or the literal meaning from the source language but ignores product localization to the target consumers. Functionalism, as a theory, which best deals with the equifunctional translation, can serves as a general guide to product instruction translation.

\section{The Skopos Theory in Translation}

Skopos is a Greek word for "aim" or "purpose" and was introduced into translation theory in the 1970 s by Hans J. Vermeer as a technical term for translation. The general theory elaborates in detail in the book co-authored by Vermeer and Reiss in 1984.

According to Skopostheorie, the prime principle determining any translation process is the purpose of the overall translation action [4]. In Vermeer's point of view, three possible kinds of purpose in the field of translation are: the general purpose aimed at by the translator in the translation process, the communicative purpose aimed at by the target text in the target translation and the purpose aimed at by a particular translation strategy or procedure.

The top-ranked rule for any translation is thus the Skopos rule, in Vermeer's words," the end justified the means". Vermeer gives an explanation about the Skopos rule in the following way: Each text is produced for a given purpose and should serve this purpose. The Skopos rule thus reads as follows: translate/interpret/speak/write in a way that enables your text/translation to function in the situation in which it is used and with people who want to use it and precisely in the way they want it to function [8]

The Skopostheorie, as the core of the Functionalism, means all the aspects of a translation are decided by the general purpose of a translation. Its translation principles details in three rules. The Skopos rule, which holds that the general Skopos determines a translational action. The coherence rule, which specifies that the translated text must be acceptable with the receivers' situation and meet the norms of the local culture. In addition, the fidelity rule, which concerns the degree of faithfulness to the original text, in that order in terms of importance. Among these three rules, the 
fidelity rule is subordinate to the coherence rule, and these two rules ultimately depend on the Skopos rule. [1]

These three rules can be used as general guidance for translation, but as a specific field of translation, instruction translation needs to have its own principles, since it has some unique features compared with other translation fields.

\section{Translation Principles for Product Instruction}

Functionalism is not only "the purpose of the pursuit of text type", what is more important is that the purpose of the pursuit of communication or translation of the purpose. [2] Even though a translator may infer the possible function from the given source text with his previous experience, however, we have to consider the situational and cultural, etc. differences between the source and target texts. To find the aspects in which the source and target texts may diverge, a translator should compare the source text with the expected translation defined in the translation brief, which should give the following information:

The intended text functions;

The target text addressees;

The time and place of text reception;

The medium over which the text will be transmitted;

The motive for the production or reception of the text.

Translators can choose literal translation or free translation according to their different purposes of translation. Toward other translation strategies and methods, the Functionalism theory is more objective and tolerant. Any translation strategy, no matter it is Nida's translation dynamic and formal translation, Peter Newmark's semantic and communicative translation, or the traditional Chinese faithfulness, expressiveness and elegance, can be justified if it realizes the translating purpose. Reiss said, "Any translation type (such as word-for-word translation, literal translation or learned translation) may be justified in particular circumstances for a particular translation aim"[7]

The intended function of instruction translation is to inform the target readers what a certain product and how to use it correctly. The Functionalism theory believes faithfulness is decided both by the translator's interpretation of the source text and on the translation purpose. According to the purpose of instruction translation, hiding or missing any information of the source instruction will mislead the user and sometimes even cause dangers. Thus, translators must be faithful and loyal to transfer the information of original instructions.

In Nord's model of basic text functions, the major function of instructions is referential, involving "reference to objects and phenomenon of the world or of a particular world, perhaps a fictional one" [4]. To carry out this function, the receiver must be able to coordinate the message with his model of the particular worlds involved. Since cultural perspectives and traditions determines word models, receivers in the source culture may interpret the referential differently from those in the target culture. A translator must adhere to the coherence rule and localize these differences so that the translated text can be accepted in the target culture.

In order to produce a translated instruction which can realize the writer's original purposes; which is faithful and without any cultural barriers to the source text, the following three principles should be obeyed:

\section{To realize the original purposes of the source instruction.}

The top-ranking rule of the Skopostheorie is the Skopos rule, which means the translation purpose or purposes decide all the other aspects of translating action. Before translating an instruction, a translator had better have an in-depth and thorough reading and then analyze it with the following questions:

What kind of instruction is it?

What are the contents of the instruction?

What are the language features and text types?

What are the purposes of the original writer?

Who will be the target reader? 
What translation principles and methods can be applied?

Only after finding the answers to these questions can the translator have a start. While translating the instruction, the translator should always bear the purposes of the source texts in mind and enable the translation to function in the target language situation in which it is used and with consumers who want to use it and precisely in the way they want it to function.

To Be Faithful to the Original Instruction. Though the Skopostheorie put fidelity in the third place, it plays a key role in instruction translation, because of the specific purpose of instruction translation.

First from morality, faithfulness means a translator must be faithful to the source text, even under special requirements of the translation commissioner. For example, a cosmetic manufacturer, considering the sales of the product, asks the translator to exaggerate the effects of the cosmetics. Under this requirement, the translator must choose faithfulness, no matter how much is paid. Because a translator must be responsible for his translation and make sure that his/her translated, work does not have any falsehood or exaggeration. This is what Nord called loyalty.

Second, the translation must be accurate and complete. Every translator pursues accuracy and completeness, but limited by one's language ability, otherwise, the original goals are very hard to obtain. One's proficiency decides that whether he can completely pass the source text information. Sometimes it even causes wrong translations. Translators should keep improving his/her technical knowledge of the field which he/she is always in to achieve the goal of faithfulness and loyalty. According to Nida, the use of a "closest natural equivalence" to reproduce the source language information, so that the target text of the original reader to the original text to understand and appreciate the original way to understand and appreciate the translated text. [4]

To Localize Cultural Differences in the Target Language. Functional translation theory emphasizes translation as a kind of "cultural transfer", a "communicative interaction", a "cross-culture event", the recipient of multi-party professionals to participate in the overall complexity of the collective behavior. [6] The Functionalism theory believes that the translated text must be coherent with the receiver's situation. That means the translated text should not contain cultural barriers. One of the most important factors in determining the purpose of translation is the audience - the intended recipient of the translation. They have their own cultural background, expectation of translation and communication needs. [10]

In the process of translation, the interaction between the original author, the translator (or the principal), the translator and the target reader is inevitable. This interaction is by no means limited to the transcoding process at the linguistic level, but rather a higher level of cultural transformation that focuses on the interaction between the original sender and the target recipient. [9] In the translation of instructions, cultural barriers exist; it demands translators' attention, because they may result in misunderstandings and even serious dangers. Nida said, "The role of language within a culture and the influence of the culture on the meanings of words and idioms are so pervasive that scarcely any text can be adequately understood without careful consideration of its cultural background. [3]

Culture is defined by Vermeer as "the entire setting of norms and conventions an individual as a member of his society must know in order to be 'like everybody' — or to be able to be different from everybody"[8]. The definition clearly shows that the core of culture is the differences. Nowadays, translation training has already changed from a simple linguistics teaching model to a blend of cultural factors and linguistics teaching model. The German Functional Translation School has been a pioneer in these two aspects for many years. [5]

Culture differences exit not only between countries and countries, but also between different areas of a country. Take the mainland and Taiwan as an example: the language of Taiwanese is also Chinese, but it is the Transitional Chinese, which indeed has some differences. First, the characters are different. It is difficult for Taiwanese to read the Simplified Chinese and for young mainlanders to read the Traditional Chinese. Second, terms in many fields are different as the separation of Taiwan for more than half a century and the culture communication barriers set by the Taiwan local government. 
Translators have to pay special attention to these term differences during the process of localization since they have different "names" in the Chinese mainland and Taiwan. On the other hand, as we know that the hardware industry in Taiwan is booming, there are many world famous manufacturers like ASUS, MSI, Gigabyte, AOC etc., most of their products have both the Simplified and Traditional Chinese version instructions, but some other companies only provide the same instructions just like the local consumers in order to reduce cost. These companies do not realize how important of the localization of instructions is, since the traditional version instructions leave great difficulties to the mainland consumers to follow. The so-called cost-saving actions caused many complaints thus leaves the consumers a feeling that the product is not so professional and at least it does not have the interest of the consumers in mind so much. Therefore, translators need to know that a well-localized translation version of instructions is a good way to maintain consumers, so try to remove culture barriers is an important task in instruction translation.

\section{Conclusion}

The importance of instruction translation becomes more and more evident with the economic globalization. Any corporation that wants to open the oversea market or enhances their business abroad should pay enough attention to instruction translation, since a lame instruction version makes no sense to consumers, although the product may be excellent.

Based on the Skopos theory, the core theory of Functionalism, this thesis tries to study the translation of English instructions, to find the appropriate translation principles. I hope the findings of this thesis would be helpful and would work as a general guide for product instruction translation.

This thesis aims to provide a practical guide for instruction translation practice, improve the quality of translation and produce translated instructions, which are accepted and respected by the target society.

As a general study of instruction translation, which focuses on common problems of all instructions, the translation principles and methods may be somewhat not deep for concrete and specific instruction translation fields, like food, medicine, electronic appliances instructions. The features of these instructions are different to some extent and worth a deeper and more comprehensive research.

Future research could take more consideration in aspects, such as the grammatical difference between English and Chinese instructions and try to analyze it from the point view of Communicative theory, which is also an appropriate theory for instruction translation.

\section{References}

[1] Fangyi Hu Journal of Jishou University (Social Sciences Edition) Vol. 31 (2010) No.03, p.172 (In Chinese)

[2] Guangrong Li Reflection on the Misunderstanding and Misuse of the Functionalism Journal of Tianjin Foreign Studies University (2010) Vol. 17 No.1, p.43 (In Chinese)

[3] Nida Eugene: Language and Culture (Shanghai Foreign Education Press, China 2001) p.1

[4] Nord Christiane: Translating as a Purposeful Activity: Functionalist Approaches Explained (Shanghai Foreign Language Education Press China 2001) p.40-45,86, 91

[5] Gentzler Edwin. Contemporary Translation Theories (Revised Second Edition) (Shanghai Foreign Education Press, China 2004) p.70

[6] Snell-Hornby: Translation Studies, An Integrated Approach (Shanghai Foreign Education Press. China 2001) p.47

[7] Reiss Katharina: Translation Criticism: the Potentials and Limitations (Shanghai Foreign Language Education Press China 2004) p.54 
[8] Vermeer Hans. Skopos and Commission in Transitional Action: The Translation Studies Reader. Routledge England 2000) p.28, 173

[9] Wenbo Jia Shanghai Journal of Translators (2007) No.2, p9 (In Chinese)

[10]Zuoyou Hu Academics in China, Vol. 133 (2008) No.06, p.252. (In Chinese) 